\title{
Extricating erbium
}

Claude Piguet reflects on the history of erbium, which is very much intertwined with its rare earth cousins yttrium, ytterbium and terbium.

$\mathrm{T}$ he discovery of erbium did not come in a single 'eureka' moment, but rather in a series of steps, inextricably linked to the discovery of more new elements than the residents of one small town in Sweden could have ever anticipated. It started in 1787 when a lieutenant in the Swedish Royal Army named Carl Arrhenius collected a curious black stone in the town of Ytterby, ten miles away from Stockholm. Chemical analysis of the mineral revealed that it contained a new earth (a metal oxide, in modern terminology, but considered an element at the time), later named yttria after the town in whose quarry it was found.

Concurrently, in France, the revolutionary policy that 'La République n'a pas besoin de savants ni de chimistes' (the French republic needs neither scientists nor chemists) sent Lavoisier to the guillotine, his discovery of oxidation reactions not enough to spare him. It was, however, enough to force his contemporaries to reconsider the concept of an element, abandon phlogiston theory and investigate the extraction of metallic elements from oxides. Back in Sweden, Carl Mosander applied newly developed reduction techniques to transform the oxide yttria into yttrium. Only then was it realized that this 'single species' contained not only yttria (a colourless oxide), but also two other oxides that could be isolated from the original sample: a yellowish substance that he named erbium oxide and a pale amethystcoloured powder he called terbium oxide.

Pushed by his mentor for rapid dissemination of his results, Mosander published the discovery of erbium and terbium in 1843 , but with severe reservations concerning the purity of these two new elements ${ }^{1}$. Submitted to systematic investigations by a dream team of chemists, geologists and spectroscopists in Geneva over the next few decades, Mosander's erbium and terbium proved to be mixtures of no fewer than seven elements: not only

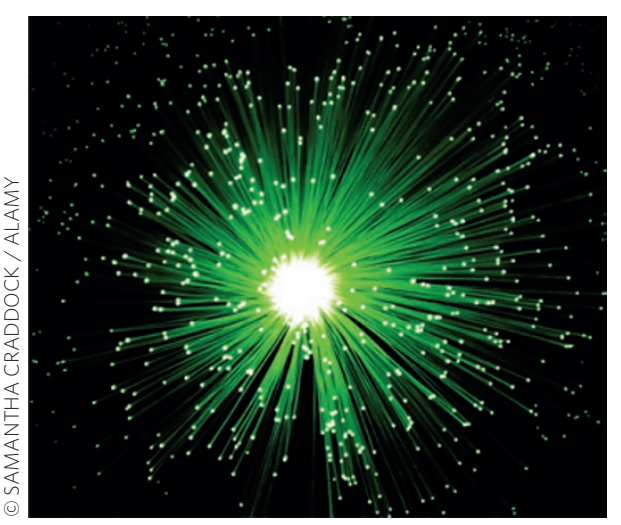

erbium and terbium, but also ytterbium, scandium, thulium, holmium and gadolinium. Ytterby had turned out to be quite the element mine, with four elements eventually named after it, out of eight new elements extracted from minerals collected there. The difficulty in separating these elements from each other was further underlined by the simultaneous extraction, in 1907, of yet another element from a sample of ytterbia: lutetium, or cassiopeium, depending on whether one is a turn-of-thecentury French or German chemist.

Unequivocal proof for the existence of the elements yttrium, terbium and erbium was obtained by optical spectroscopy in 1864. In one final twist, however, the spectroscopist Delafontaine, in confirming their existence, erroneously switched Mosander's original names and called the amethyst-coloured compound erbium oxide and the pale yellow substance terbium oxide. This historical reversal has been carried through until the present day, so what we now know as the sesquioxide $\mathrm{Er}_{2} \mathrm{O}_{3}$ is characterized by a pale pink colour, rather than the yellow that Mosander would have recognized as erbium oxide.

The reconciliation of atomic theory with the periodic table at the turn of the twentieth century finally placed erbium and its fellow lanthanides in the 4 -block, after which erbium research dwindled for fifty years. In 1959, however, interest in erbium was reawakened in association with the nascent field of photonics. The plethora of regularly spaced and long-lived excited states found in $\mathrm{Er}^{3+}$ resulted in it becoming the perfect candidate for an experimental demonstration of a theoretical infrared detector, in which photons could be detected and counted through sequential absorptions in the successive excited levels of a given ion in a solid - that is, using superexcitation as a photon detector ${ }^{2}$.

While the proposed upconversion pathway strictly relied on linear absorption from the ground state through successive excited states for detection, the direct absorption of photons by an $\mathrm{Er}^{3+}$ ion remained inefficient. A breakthrough came in 1966, when François Auzel demonstrated that superexcitation could greatly benefit from indirect light harvesting carried out by partner ions, followed by energy transfer to an erbium activator ${ }^{3}$, a mechanism similar to that operating in erbium lasers, which are used today in dentistry and some skin treatments.

Returning to upconversion, the introduction of small amounts of trivalent ytterbium impurities into erbium-containing solids is now exploited for the design of highly efficient upconverters of near-infrared to green light used in laser pointers, in solar cell technologies and as dopants for visiblelight-emitting optical fibres (see illustration). Given the great ingenuity and effort required to separate and purify these elements by early chemists, the fact that we now willingly contaminate erbium with ytterbium is, perhaps, somewhat ironic.

\section{CLAUDE PIGUET is in the Department} of Analytical and Inorganic Chemistry, University of Geneva, CH-1211 Geneva 4, Switzerland.e-mail: claude.piguet@unige.ch

\footnotetext{
References

1. Szabadvary, F. in Handbook on the Physics and Chemistry of Rare Earths Vol. 11 (eds Gschneidner, K. A. Jr \& Eyring, L.) 33-80 (Elsevier, 1988).

2. Auzel, F. Chem. Rev. 104, 139-173 (2004).

3. Auzel, F. C. R. Acad. Sci. Paris B262, 1016-1019 (1966).
} 